\title{
BMJ Global Health Efficacy of three feeding regimens for home-based management of children with uncomplicated severe acute malnutrition: a randomised trial in India
}

Nita Bhandari, ${ }^{1}$ Sanjana Brahmawar Mohan, ${ }^{1}$ Anuradha Bose, ${ }^{2}$ Sharad D lyengar, ${ }^{3}$ Sunita Taneja, ${ }^{1}$ Sarmila Mazumder, ${ }^{1}$ Ruby Angeline Pricilla, ${ }^{2}$ Kirti Iyengar, ${ }^{3}$ Harshpal Singh Sachdev, ${ }^{4}$ Venkata Raghava Mohan, ${ }^{2}$ Virendra Suhalka, ${ }^{3}$ Sachiyo Yoshida, ${ }^{5}$ Jose Martines, ${ }^{6}$ Rajiv Bahl, ${ }^{5}$ for the Study Group

To cite: Bhandari N, Mohan SB, Bose A, et al. Efficacy of three feeding regimens for home-based management of children with uncomplicated severe acute malnutrition: a randomised trial in India. BMJ Global Health 2016;1:e000144. doi:10.1136/bmjgh-2016000144

- Additional material is published online only. To view please visit the journal online (http://dx.doi.org/10. 1136/bmjgh-2016-000144).

Received 30 July 2016 Revised 30 November 2016 Accepted 1 December 2016

CrossMark

For numbered affiliations see end of article.

Correspondence to

Dr Nita Bhandari;

nita.bhandari@sas.org.in

\section{ABSTRACT}

Objective: To assess the efficacy of ready-to-use therapeutic food (RUTF), centrally produced RUTF (RUTF-C) or locally prepared RUTF (RUTF-L) for homebased management of uncomplicated severe acute malnutrition (SAM) compared with micronutrientenriched (augmented) energy-dense home-prepared foods (A-HPF, the comparison group).

Methods: In an individually randomised multicentre trial, we enrolled 906 children aged 6-59 months with uncomplicated SAM. The children enrolled were randomised to receive RUTF-C, RUTF-L or A-HPF. We provided foods, counselling and feeding support until recovery or 16 weeks, whichever was earlier and measured outcomes weekly (treatment phase). We subsequently facilitated access to government nutrition services and measured outcomes once 16 weeks later (sustenance phase). The primary outcome was recovery during treatment phase (weight-for-height $\geq-2 \mathrm{SD}$ and absence of oedema of feet).

Results: Recovery rates with RUTF-L, RUTF-C and A-HPF were $56.9 \%, 47.5 \%$ and $42.8 \%$, respectively. The adjusted OR was $1.71(95 \% \mathrm{Cl} 1.20$ to 2.43 ; $\mathrm{p}=0.003)$ for RUTF-L and $1.28(95 \% \mathrm{Cl} 0.90$ to 1.82 ; $\mathrm{p}=0.164$ ) for RUTF-C compared with A-HPF. Weight gain in the RUTF-L group was higher than in the A-HPF group (adjusted difference $0.90 \mathrm{~g} / \mathrm{kg} /$ day, $95 \%$ $\mathrm{Cl} 0.30$ to $1.50 ; \mathrm{p}=0.003)$. Time to recovery was shorter in both RUTF groups. Morbidity was high and similar across groups. At the end of the study, the proportion of children with weight-for-height Z-score (WHZ) >-2 was similar (adjusted OR 1.12, 95\% $\mathrm{Cl} 0.74$ to $1.95 ; \mathrm{p}=0.464)$, higher for moderate malnutrition ( $\mathrm{WHZ}<-2$ and $\geq-3$; adjusted $\mathrm{OR} 1.46$, $95 \% \mathrm{Cl} 1.02$ to 2.08; $\mathrm{p}=0.039$ ), and lower for those with SAM (adjusted OR $0.58,95 \% \mathrm{Cl} 0.40$ to 0.85 ; $p=0.005)$ in the RUTF-L when compared with the A-HPF group.

Conclusions: This first randomised trial comparing options for home management of uncomplicated SAM

\section{Key questions}

What is already known about this topic?

- Pooled analysis of three quasi-randomised controlled trials in Malawi, which evaluated ready-to-use therapeutic food (RUTF) in the home management of children with uncomplicated severe acute malnutrition (SAM) compared with standard diets, revealed that RUTF was associated with higher recovery rates (risk ratio $1.32,95 \% \mathrm{Cl} 1.16$ to 1.50 ).

- A Cochrane review concluded that given the limited evidence, it is not possible to reach definitive conclusions regarding differences in clinical outcomes in children with SAM who were given RUTF compared with standard diets.

What are the new findings?

- This first randomised trial comparing RUTF with energy and nutrient-dense home-prepared foods (the comparison group) confirms the efficacy of RUTF in the treatment of children with uncomplicated SAM.

- The study provides insights on the importance of feeding efforts and the caregiver support required for higher efficacy and highlights the importance of adequate continued inputs after initial treatment to sustain the benefits.

Recommendations for policy

- Children with uncomplicated SAM can be managed at home with RUTF instead of through inpatient hospitalisation.

confirms that RUTF-L is more efficacious than A-HPF at home. Recovery rates were lower than in African studies, despite longer treatment and greater support for feeding.

Trial registration number: NCT01705769;

Pre-results. 


\section{INTRODUCTION}

Severe acute malnutrition (SAM), defined as weight-for-height Z-score (WHZ) $<-3$ SD, markedly increases risk of mortality in under-5 children. ${ }^{1}$ Annually, 0.5-2.0 million deaths are attributed to SAM. ${ }^{2}$ Of the 20 million children with SAM worldwide, over eight million are from India, where around 5\% of under-5 children suffer from SAM. ${ }^{2-4}$

With a standardised hospital-based management protocol proposed by the $\mathrm{WHO}$, recovery rates of around $80 \%$ have been reported and case fatality rates ranged between $3.4 \%$ and $35 \% .^{5-7}$ A very small proportion of children suffering from SAM receive effective management in India. Families seek medical care only when children with SAM have complications. They are reluctant to accept long hospital stay due to, for example, loss of wages and no arrangements at home to take care of other siblings. Perceptions that the disease is not severe enough to warrant long hospitalisation, the fear of hospitals, past experiences that were unpleasant and the cost of hospital care are also contributory factors . ${ }^{2}$ 8-10

Home-based management after initial hospitalisation was proposed for children with SAM as an effective strategy to increase coverage. ${ }^{10}$ This was supported by the development of ready-to-use therapeutic food (RUTF) ${ }^{8}{ }^{11}$ Studies in Africa showed that, for homebased management of uncomplicated SAM, RUTF achieved recovery rates similar to those with hospitalbased management. ${ }^{12-16}$

Since 2007, the WHO recommends RUTF for homebased management of uncomplicated SAM. ${ }^{17}$ However, acceptance of this recommendation has been limited in countries like India. An important reason for the reluctance is the lack of evidence from controlled trials of the efficacy of RUTF compared with other treatment options. Experts have also questioned the 'standardised diets' used in studies. They argue that the comparison group should be given locally produced foods high in energy and proteins with adequate micronutrients. ${ }^{18-20}$ In addition, there are questions in India about the use of commercially produced RUTF over locally produced 'analogous medical nutrition therapy' or augmented home foods. Locally produced RUTF using indigenous foods may be less expensive and more sustainable if its efficacy could be proved. ${ }^{19}$ Reviews, including the most recent Cochrane review (2013), recommend welldesigned, adequately powered, pragmatic randomised trials to compare treatment options for home-based management of uncomplicated SAM. ${ }^{19}{ }^{21}$ A policy review in India reached a similar conclusion. ${ }^{22}$

We therefore conducted a randomised trial to compare the efficacy of centrally produced RUTF (RUTF-C) and locally prepared RUTF (RUTF-L) for home-based management of children with uncomplicated SAM on recovery rates compared with micronutrient-enriched (augmented) energy-dense home-prepared foods (A-HPF), the comparison group.
METHODS

\section{Study design}

In this randomised trial, children aged 6-59 months with uncomplicated SAM were randomised into one of the three groups: RUTF-C, RUTF-L and A-HPF.

The primary outcome was recovery (defined as WHZ $\geq-2 \mathrm{SD}$ of the WHO standards and absence of oedema of feet) by 16 weeks after enrolment. ${ }^{23}$ Secondary outcomes included weight gain, time to recovery, prevalence of diarrhoea, acute lower respiratory tract infection (ALRI) and fever, mortality and hospitalisations during the treatment phase (until recovery or 16 weeks after enrolment, whichever was earlier). Another secondary outcome was the proportion of children with $W H Z \geq-2 \mathrm{SD}$ at the end of the sustenance phase (16 weeks after completion of the treatment phase). The cost of the feeding regimens, the families' and health workers' perceptions about the regimens, and the factors that affect recovery (other secondary outcomes) will be published in separate manuscripts.

\section{Study sites}

We conducted the study in three diverse geographical settings in India-Rajasthan, Tamil Nadu and Delhi. The study populations were low-income households quite diverse, with a mix of rural and urban areas. The three sites also varied in the programmatic context. Enrolment began in October 2012 and follow-up was completed in April 2015.

\section{Study oversight}

The study was approved by the Government of India and the state governments. Oversight was provided by the National Research Alliance for SAM established by the Indian Government. An independent Data Safety Monitoring Board (DSMB) periodically reviewed the study and provided recommendations.

\section{DSMB recommendations}

In the original proposal, the period of treatment and measurement of the primary outcome were set at 8 weeks. This choice was based on the findings of studies in Africa that showed that most children with SAM recovered within 8 weeks of starting RUTF. ${ }^{12} 2425$ Based on a a priori decision, the DSMB reviewed the study after the initial 20 enrolments. They recommended that the intervention be given for a maximum of 16 weeks, instead of the 8 weeks described in the initial protocol. The justification was that although recovery rates by 8 weeks were low, the intervention, if effective over a period of 16 weeks, would still be of considerable public health interest.

Additional suggestions were recommended during a DSMB review conducted when about $40 \%$ children had been enrolled. These included increasing the sample size and strengthening the support for feeding as recovery rates among enrolled children were still low. In response, peer supporters who would help caregivers to 
feed their children were identified at all sites and the sample size was increased.

\section{Sample size}

The study involved two comparisons: between RUTF-C and A-HPF, and between RUTF-L and A-HPF. A-HPF was the comparison group. When we planned this study, published non-randomised studies conducted in Africa had shown 17-23\% difference in recovery between RUTF and a standard diet. ${ }^{12} 1526$ We therefore hypothesised a conservative $15 \%$ difference between the RUTF and the comparison group (A-HPF) for calculation of sample size. Assuming an $80 \%$ recovery in the intervention group and $65 \%$ in the comparison group (ie, a $15 \%$ difference) with $90 \%$ power and $\alpha=0.025$, we required 231 children in each group. We increased it by $10 \%$ to account for loss of follow-up resulting in the original sample size estimate of 765 . When the overall recovery rate was observed to be lower than assumed, the DSMB recommended an increase in sample size to at least 900 children to preserve the power to test the a priori hypothesis of $15 \%$ difference between the intervention and comparison groups.

\section{Study procedures}

Identification of children with SAM

We conducted a door-to-door survey in the defined study populations to identify all children aged 659 months. After written informed consent from the caregiver, the mid-upper arm circumference (MUAC) was measured (Chasmors CTM03 tape; accuracy $1 \mathrm{~mm}$ ). Children with MUAC $<130 \mathrm{~mm}$ were brought to the study clinic. At the clinic, weight (Seca 385 digital weighing scale; accuracy $20 \mathrm{~g}$ ) and height/length (Seca 417 infantometer for length, Seca 213 stadiometer for height; accuracy $1 \mathrm{~mm}$ ) were measured, and oedema of feet checked. The WHZ were calculated using the WHO Growth Standards. ${ }^{27}$ The WHO Anthro software was used for calculating WHZ (http://www.who.int/ childgrowth/software/en/). Children with $\mathrm{WHZ}_{<-3} \mathrm{SD}$ or oedema of feet, or both were identified as SAM. ${ }^{17} 23$ Children with complications were followed up and considered for enrolment after improvement.

\section{Screening and enrolment}

Physicians screened all children with SAM for signs of severe illness based on the Integrated Management of Neonatal and Childhood Illness (IMNCI) algorithms. ${ }^{28}$ Haemoglobin was estimated (HemoCue method) and appetite tested using RUTF-C as the test feed. Children with severe illness requiring hospitalisation, allergy to milk and haemoglobin $<6 \mathrm{~g} / \mathrm{dL}$, and who were unable to consume the test feed were considered to have complicated SAM and were taken to health facilities for hospital management. The remaining children with uncomplicated SAM, whose families were likely to remain in the study area over the next 4 months, and whose parents gave written informed consent were enrolled. Children with a sibling previously enrolled in the study were excluded. MUAC, triceps and subscapular skinfold thickness (Holtain skinfold callipers; accuracy $0.2 \mathrm{~mm}$ ) of enrolled children, and weight and height of mothers were also measured.

\section{Allocation and concealment}

A WHO statistician, not otherwise involved with the study, prepared randomisation lists. Randomisation was stratified by site and age categories (6-17 and 1859 months) using block sizes of variable length (3, 6 or 9). Allocation into study groups was concealed using Serially Numbered Opaque Sealed Envelopes (SNOSE) prepared by the WHO. The allocation ratio was 1:1:1 and the children were recruited in all three groups according to the randomisation list. The SNOSE next in sequence was opened only after completing an enrolment.

\section{Interventions during treatment phase}

We delivered foods free of cost in the three study groups, with the aim of ensuring an intake of at least $175 \mathrm{kcal} / \mathrm{kg}$ body weight/day.

The composition of RUTF-C, packaged in $92 \mathrm{~g}$ sachets (Compact India, Gurgaon, India), conformed to the WHO recommendations. ${ }^{17}$ Each site team was trained in the preparation of RUTF-L by a consultant who had participated in the African studies. The preparation was carried out under stringent conditions that included controlled temperature and humidity, restricted access to the preparation room, hygienic conditions, periodic pest control and good ingredient quality. RUTF-L was prepared in a designated room by trained staff and packaged in transparent food grade $250 \mathrm{~g}$ jars. ${ }^{29}$ The composition of RUTF-L was similar to the one used in African research studies and programmes, and conformed to the WHO recommendations (table 1).

Microbiological testing of RUTF-L was performed every 3 months. Samples were sent to an external accredited laboratory and tested for aflatoxin content, Escherichia coli, coliform count, yeast, moulds, pathogenic Staphylococci, Salmonella, Listeria monocytogenes, Enterobacter and Clostridium perfringens.

Families of children in the comparison group (A-HPF) were given raw ingredients to prepare foods. These included locally available and acceptable cereals and pulses, sugar, oil, milk and eggs. Recipes for making energy-rich and nutrient-rich foods for children were promoted. A micronutrient preparation providing the recommended daily intake of vitamins and minerals for a child with SAM was given to caregivers, to be added to the cooked meal prior to feeding. ${ }^{23}$ In the A-HPF group, we gave food ingredients in excess of requirements for the child given the expectation of some sharing within the family. In all the three groups, we aimed to achieve intakes of $175 \mathrm{kcal} / \mathrm{kg}$ body weight/ day for the enrolled child. 
Table 1 Composition and appearance of RUTF-C and RUTF-L

\begin{tabular}{lll}
\hline Description & RUTF-C & RUTF-L \\
\hline Energy (kcal)/100 g & 543 & 528 \\
\hline Nutrient content $(\mathrm{g})$ & & \\
$\quad$ Proteins & 15 & 15 \\
\hline Lipids & 34.8 & 33 \\
\hline Carbohydrates & 43.5 & 46 \\
\hline Ingredients & & \\
\hline Peanut paste & $30 \%$ & $26 \%$ \\
\hline Sugar & $29 \%$ & $27 \%$ \\
\hline Milk solids & $20 \%$ & $25 \%$ \\
\hline Vegetable oil & $18 \%$ & $20 \%$ \\
\hline Mineral mix & Identical* & Identical \\
\hline Vitamin mix & Identical $\dagger$ & Identical† \\
\hline Emulsifier & Yes & No \\
\hline Antioxidant & Yes & No \\
\hline Consistency & Thicker and sticky & Thinner \\
\hline Texture & Smooth & Granular \\
\hline
\end{tabular}

*Minerals per $100 \mathrm{~g}$ : calcium $400 \mathrm{mg}$, phosphorus $400 \mathrm{mg}$ potassium $1100 \mathrm{mg}$, magnesium $110 \mathrm{mg}$, sodium <290 mg, iron $10 \mathrm{mg}$, zinc $12 \mathrm{mg}$, copper $1.5 \mathrm{~m}$, iodine $100 \mu \mathrm{g}$, selenium $30 \mu \mathrm{g}$. †Vitamins per $100 \mathrm{~g}$ : vitamin $\mathrm{A} 0.9 \mathrm{mg}$, vitamin $D_{3} 18 \mu \mathrm{g}$, vitamin $\mathrm{K}$ $21 \mu \mathrm{g}$, vitamin E $27 \mu \mathrm{g}$, vitamin C $54 \mathrm{mg}$, vitamin $B_{1} 0.5 \mathrm{mg}$, vitamin $B_{2} 1.8 \mathrm{mg}$, vitamin $B_{6} 0.7 \mathrm{mg}$, vitamin $B_{12} 1.6 \mu \mathrm{g}$, niacin $5.8 \mathrm{mg}$, Ca-D pantothenate $3 \mathrm{mg}$, folic acid $225 \mu \mathrm{g}$, biotin $70 \mu \mathrm{g}$.

RUTF-C, centrally produced ready-to-use therapeutic food; RUTF-L, locally prepared ready-to-use therapeutic food.

During the treatment phase, household visits were carried out by a study worker with similar educational qualifications to the government's Accredited Social Health Activist (ASHA) who are women from the communities trained as health educators and promoters by the government, and a key cadre in India's community health worker programme designed to improve maternal and child health. The visits were conducted weekly to counsel, resolve caregivers' queries and difficulties, to replenish supplies, and to collect empty RUTF-L jars and wrappers of RUTF-C packets. ${ }^{30}$ The workers used site-specific counselling cards made in the local language. The cards included messages on how frequently, how much and how to feed, on continuing breast feeding, good hygiene and advice on family meals. A copy of the card was also given to the family. In the A-HPF group, local, culturally appropriate, energy-rich and nutrient-rich recipes were also printed on the cards. Information on recommended recipes is provided in the online supplementary file. Information on intake of RUTF-L and RUTF-C was recorded; the amounts of ingredients used weekly were documented for the A-HPF group. For calculation of food consumption, the used packets (RUTF-C) or jars (RUTF-L) were collected from each home during the weekly visit throughout the treatment phase. The total calories consumed were calculated by multiplying the number of calories in a packet (500 kcal for RUTF-C) and jar (1328 kcal for RUTF-L) by the number of packets or jars consumed by the child. The calorie consumption in $\mathrm{kcal} / \mathrm{kg} / \mathrm{day}$ was calculated by dividing the total calories consumed by the number of days the child was available during the treatment phase and weight at enrolment. We did not collect consumption data for the comparison group given the greater difficulty in capturing valid information. The neighbourhood peer support workers recruited post-DSMB recommendations visited homes several times a day to help caregivers feed their children in all the three groups. They were given a daily financial incentive for this activity.

\section{Cointerventions in the treatment and sustenance phases}

Co-interventions were similar in the three groups. At enrolment, all children received oral amoxicillin for 5 days. Those aged 2 years or older were given antihelminthics (mebendazole) for 3 days. Children with anaemia (haemoglobin $\geq 6$ to $\leq 11 \mathrm{~g} / \mathrm{dL}$ ) in the A-HPF group were given iron and folic acid. A mega dose of vitamin A was given to children with signs or symptoms of vitamin A deficiency. Immunisation was facilitated according to the National Immunisation Schedule. Sick children visiting the study clinics were treated following the IMNCI guidelines. ${ }^{28}$

During follow-up, sick children attending the study clinic or referred by workers during home visits were treated by physicians, and those with severe illness were referred to hospitals. ${ }^{28}$ Hospitalised children were treated according to local policy. Transport and treatment were provided free of cost. The intervention was restarted after families returned home.

After completion of the treatment phase, the study team facilitated linkages between the families and the government-run anganwadi centres, where supplementary food is provided under the Integrated Child Development Services (ICDS) scheme. $^{31}$ This was carried out over the next 16 weeks (sustenance phase).

\section{Outcome measurement}

During the treatment phase, an independent outcome measurement team took weekly anthropometric measurements. This team was blinded as far as possible to the group to which the child was allocated. They measured weight, height, MUAC, skinfold thickness using equipment similar to that used at enrolment, and looked for oedema of feet. Weight-for-height was estimated using the weight and height measured on that day. Information on diarrhoea morbidity, ALRI, fever and hospitalisation was also ascertained.

During the treatment phase, children with no change or deterioration in WHZ at 4 weeks postenrolment or deterioration in WHZ for 2 consecutive weeks were taken to a paediatrician for assessment. Children who did not recover by 16 weeks were evaluated in hospitals. All hospitalisations and deaths were reported within 3 days to the site ethics committee, the WHO 
and the coordination unit that provided oversight to the study.

At the end of the sustenance phase, anthropometry data were obtained during a single visit and we asked about hospitalisations during the 16 weeks since the completion of the treatment phase, that is, the sustenance phase.

\section{Quality assurance}

All teams were extensively trained prior to study initiation and periodically thereafter. Anthropometric measurement protocols were based on the WHO recommendations; these included training in measurement techniques, periodic standardisation of teams and daily calibration of equipment. ${ }^{32}$ Two 'gold standards' (persons with several years of experience in measurement and trainers for the anthropometry team) conducted standardisation exercises at all sites every 3 months. Equipment was calibrated every day. Supervisors reviewed activities daily. Quality control visits were conducted by an independent team through directly supervised and independent revisits for at least $1 \%$ each of follow-up and outcome measurement visits.

\section{Ethical approvals}

The study was approved by the institutional ethics committees of each participating institution (Society for Applied Studies, New Delhi: SAS ERC/40/2012; Christian Medical College, Vellore: IRB-A13-19-09-2012; Action Research and Training for Health, Udaipur: ARTH IEC dated 14 January 2013) and the WHO Ethics Review Committee (Protocol ID RPC538). Written informed consent was obtained from caregivers for each different activity.

\section{Patient involvement}

The study was designed in response to a national consultation with public health researchers, clinicians, nutritionists and community leaders. The study questions and the interventions were defined during the consultation. Prior to study initiation, families in the study area were engaged by the team about effective ways of supporting mothers in home treatment with SAM for children without complications requiring hospitalisation. During the study, peer supporters from the neighbourhood supported and motivated mothers of enrolled children. Community leaders and families helped in identification of these persons. Mothers helped in the identification of ingredients and recipes to be promoted in the group of children receiving augmented home foods. The study team assisted in strengthening the families' relationship with existing nutrition services (ICDS scheme). ${ }^{31} \mathrm{~A}$ consultation was held in partnership with the national government to disseminate findings and discuss the way forward. This resulted in several states in India launching a programme for home treatment of children with uncomplicated SAM. The team provided guidance in programme design.

\section{Statistical analysis}

Data from all sites were pooled and analysed using Stata software (V.12.0). Simple comparison of means and proportions was used to check comparability between groups. In the primary analysis, we made an overall comparison of outcomes between different groups. Analysis was conducted according to intention-to-treat. All children who completed the treatment phase and for whom outcome was known were included in the analysis. We used generalised linear models to compare the efficacy of the three regimens. We adjusted for baseline characteristics where there were small non-significant differences-maternal education, caste, religion, and family structure and also for enrolment age, sex, site, peer support and the pre-enrolment WHZ.

Two-sided tests were applied. For time to recovery, Kaplan-Meier plots were prepared and a Cox proportional hazard model was run. For these analyses, all enrolled children were included until they were in the study, that is, until recovery, completion of the 16 weeks treatment phase or until they were lost to follow-up.

In addition to the primary and secondary outcomes included in the protocol, additional analyses were planned prior to completing data collection. These included comparison of anthropometric status at enrolment and at the end of the study across the groups as well as the proportion of children with SAM at the end of the sustenance phase.

\section{Definitions used}

Diarrhoea was defined as passage of three or more loose or watery stools in a 24-hour period.

ALRI was defined as presence of cough or difficult breathing and either fast breathing or lower chest indrawing.

Fever was documented based on the caregiver's report.

\section{RESULTS}

From October 2012 to September 2014, 106935 children aged 6-59 months were identified through surveys at the three sites. The $6815(6.4 \%)$ children who had MUAC $<130 \mathrm{~mm}$ were referred to the study clinics. Of the $5103(74.9 \%)$ who came to the study clinic, 1190

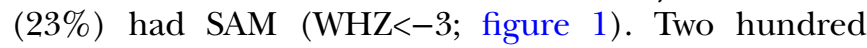
and ninety-two (24.5\%) children had a medical complication requiring referral to a hospital. They were revisited for about a month to ascertain resolution of complications; 98 of the children referred to hospital were enrolled after they recovered from the illness and were available at home. Nine hundred and six children with uncomplicated SAM were enrolled into the study (figure 1).

Eight hundred and fifty-five $(94.4 \%)$ children completed the treatment phase and 838 (92.5\%) children were measured at study completion or at the end of the sustenance phase. 
Figure 1 Trial profile. A-HPF, micronutrient-enriched (augmented) energy-dense home-prepared foods; MUAC, mid-upper arm circumference; RUTF-C, centrally produced ready-to-use therapeutic food; RUTF-L, locally prepared ready-to-use therapeutic food; SAM, severe acute malnutrition.

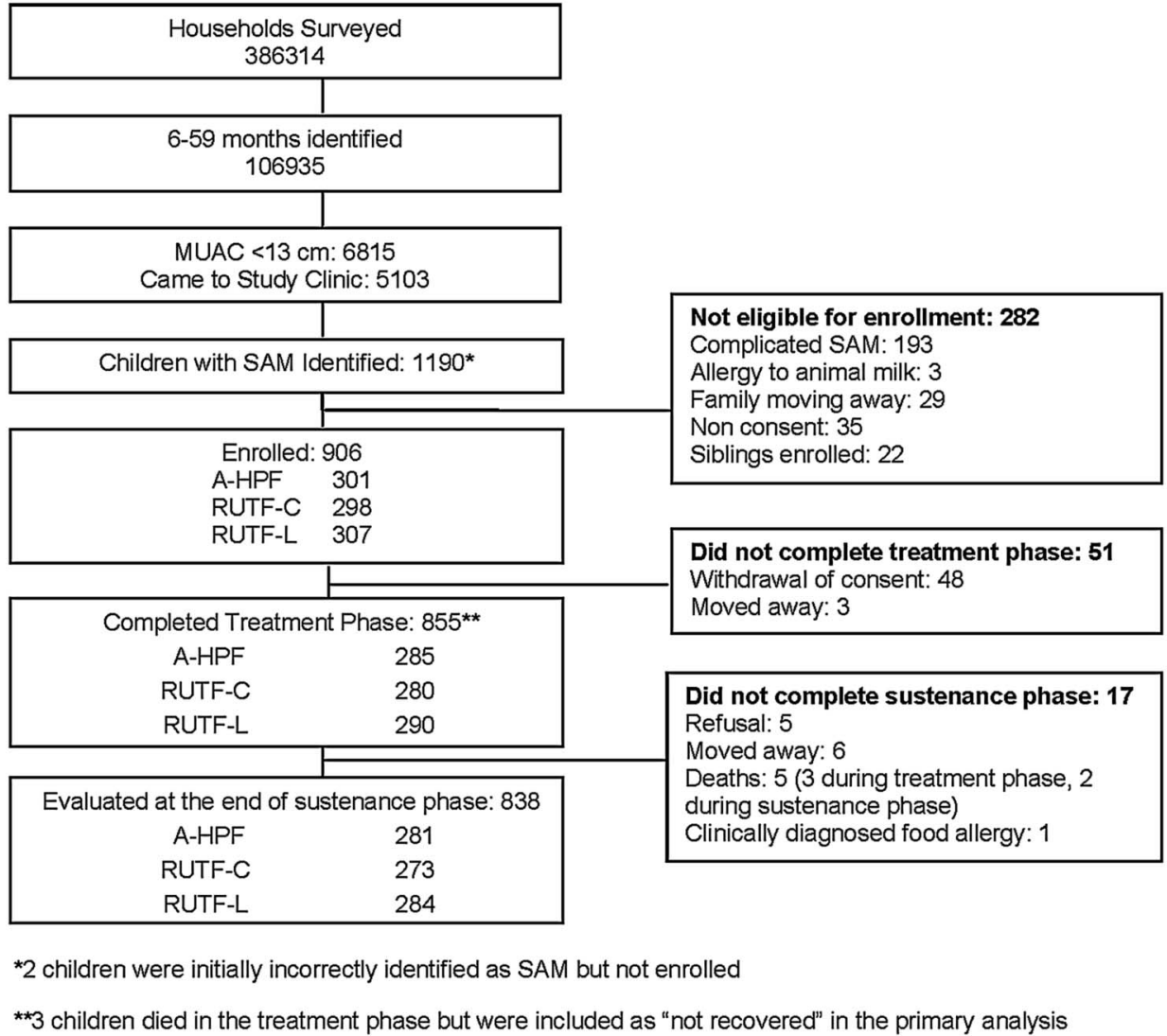

At enrolment, three-fourths of the children were stunted, $48 \%$ severely so (table 2). Only two children had oedema of feet. Almost half of the mothers had a body mass index under 18.5 (table 2).

While baseline characteristics were similar across the three groups, there were some important differences in family structure, maternal education, religion and caste.

The recovery rates with A-HPF, RUTF-C and RUTF-L were $42.8 \%, 47.5 \%$ and $56.9 \%$, respectively (table 3 ). Children in the RUTF-L group had a significantly higher rate of recovery as compared with A-HPF (adjusted OR 1.71 (95\% CI 1.20 to 2.43; $\mathrm{p}=0.003)$ ). The recovery rates in the RUTF-C group compared with the A-HPF group were OR 1.28 (95\% CI 0.90 to 1.82; $\mathrm{p}=0.164)$. The Cox proportional hazard model showed similar results. Adjusted HR compared with A-HPF group was 1.43 (95\% CI 1.13 to $1.81, \mathrm{p}=0.003)$ for RUTF-L and 1.22 (95\% CI 0.95 to $1.56, \mathrm{p}=0.112$ ) for RUTF-C.

Among children who recovered by 16 weeks, time to recovery was significantly shorter both in the RUTF-C (adjusted difference -1.34 weeks, 95\% CI -2.36 to $-0.31 ; \mathrm{p}=0.011$ ) and RUTF-L (adjusted difference -1.17 weeks, $95 \%$ CI -2.16 to $-0.17 ; \mathrm{p}=0.021$ ) groups compared with the A-HPF group.

The mean (SD) weight gain $(\mathrm{g} / \mathrm{kg} /$ day) in the A-HPF, RUTF-C and RUTF-L groups was 2.64 (3.47), 3.05 (3.41) and 3.52 (3.92), respectively.

Diarrhoea, ALRI or fever was reported by families of $520(71.5 \%)$ children. Eighty-two $(9.6 \%)$ children were hospitalised during the treatment phase. The proportion of children who were hospitalised or had diarrhoea, ALRI or fever was not significantly different across the three groups.

Recovery rates at 8 weeks after treatment are also presented as this was the original primary outcome. These were $26.6 \%, 34.8 \%$ and $40.7 \%$ in the A-HPF, RUTF-C and RUTF-L groups, respectively. The adjusted OR for the RUTF-L group compared with the A-HPF group was $1.83,95 \%$ CI 1.27 to $2.64(\mathrm{p}=0.001)$ and $1.56,95 \%$ CI 1.07 to 2.26 ( $p=0.020)$ for the RUTF-C group when compared with the A-HPF group.

The overall recovery rates in children whose families were offered peer support were substantially higher than in those who did not receive this support ( $55 \%$ vs $42 \%$, $\mathrm{p}<0.001$ ).

Kaplan-Meier curves showed that recovery started earlier in the RUTF-L group and the difference was maintained throughout the treatment phase (figure 2).

Sixteen weeks after completion of the treatment phase (sustenance phase), 838 children $(92.5 \%)$ were available for follow-up (table 4). Of these, 123 (14.7\%) met the definition of recovery, $402(48.0 \%)$ met the definition of moderate acute malnutrition $(\mathrm{WHZ}<-2$ and $\geq-3)$ and 313 children $(37.4 \%)$ had SAM (table 4$)$. The proportion of children with SAM was significantly lower in the RUTF-L group (adjusted OR 0.58 (95\% CI 0.40 to 0.85 ; $\mathrm{p}=0.005$ ); correspondingly, those with moderate malnutrition were significantly higher $(1.46,95 \%$ CI 1.02 to 2.08; $\mathrm{p}=0.039$ ) compared with the A-HPF group. The 
Table 2 Baseline characteristics of enrolled children and their families

\begin{tabular}{|c|c|c|c|}
\hline & \multicolumn{3}{|c|}{ Treatment groups } \\
\hline & A-HPF (n=301) & RUTF-C (n=298) & RUTF-L (n=307) \\
\hline Age at enrolment, mean (SD) & $25.7(14.1)$ & $24.7(13.9)$ & $25.7(14.0)$ \\
\hline Weight-for-height Z-score, mean (SD) & $-3.5(0.5)$ & $-3.5(0.4)$ & $-3.4(0.4)$ \\
\hline$<-3$ to $\geq-4$ & $266(88.4)$ & $267(89.6)$ & $282(91.9)$ \\
\hline$\geq-2$ & $75(24.9)$ & $68(22.8)$ & $67(21.8)$ \\
\hline$<-2$ to $\geq 3$ & $83(27.6)$ & $94(31.5)$ & $87(28.3)$ \\
\hline$<-3$ to $\geq-4$ & $84(27.9)$ & $82(27.5)$ & $90(29.3)$ \\
\hline$<-4$ & $59(19.6)$ & $54(18.1)$ & $63(20.5)$ \\
\hline 12.5 to 13.0 & $61(20.3)$ & $63(21.1)$ & $72(23.5)$ \\
\hline Fever/diarrhoea/cough or fast breathing) in previous 2 weeks & $159(52.8)$ & $162(54.4)$ & $166(54.1)$ \\
\hline Mother's age, mean (SD) & $27.1(5.6)$ & $27.7(5.8)$ & $27.4(4.9)$ \\
\hline Mother's $\mathrm{BMl}<18.5 \mathrm{~kg} / \mathrm{m}^{2 *}$ & $134(47.9)$ & $135(48.0)$ & $151(51.7)$ \\
\hline Mother never been to school & $117(38.9)$ & $116(38.9)$ & $136(44.3)$ \\
\hline Mother's years of schooling, median (IQR) & $5(0-9)$ & $5(0-10)$ & $5(0-8)$ \\
\hline Father never been to school & $59(19.6)$ & $66(22.1)$ & $69(22.5)$ \\
\hline Father's years of schooling, median (IQR) & $7(3-10)$ & $8(3-10)$ & $7(2-10)$ \\
\hline \multicolumn{4}{|l|}{ Religion of head of family } \\
\hline Other caste (higher class) & 59 (19.3) & $60(19.9)$ & $63(19.7)$ \\
\hline Persons residing in the house, mean (SD) & $5.9(2.4)$ & $5.7(2.5)$ & $5.9(2.4)$ \\
\hline Piped water & $55(18.3)$ & $51(17.1)$ & $57(18.6)$ \\
\hline Flush toilet within the house & $145(48.2)$ & $150(50.3)$ & $151(49.2)$ \\
\hline Facility delivery & $204(67.8)$ & $222(74.5)$ & $204(66.4)$ \\
\hline Family with below poverty line card & $97(32.2)$ & $100(33.6)$ & $105(34.2)$ \\
\hline Family structure, nuclear† & $175(58.1)$ & $196(65.8)$ & $189(61.6)$ \\
\hline
\end{tabular}

All values are $n(\%)$ unless otherwise indicated.

*BMI values not available for 53 mothers: A-HPF $(n=21)$, RUTF-C $(n=17)$, RUTF-L $(n=15)$

†Pair of adults living with their children.

A-HPF, micronutrient-enriched (augmented) energy-dense home-prepared foods; BMI, body mass index; RUTF-C, centrally produced ready-to-use therapeutic food; RUTF-L, locally prepared ready-to-use therapeutic food.

WHZ between enrolment and the end of the sustenance phase improved for all enrolled children. However, the improvement appears to be greater in the RUTF-L group compared with the A-HPF group (difference in mean Z-scores $0.11,95 \%$ CI 0.0 to $0.22, \mathrm{p}=0.051$; table 4).The mean (SD) amount of RUTF-L consumed was 193.27 (94.03) g/day and RUTF-C 172.83 (89.10). The mean (SD) $\mathrm{kcal} / \mathrm{kg} /$ day consumed was 140.19 (65.41) and 129.69 (65.09), respectively. Consumption was not measured in the A-HPF group.

\section{DISCUSSION}

This is the first randomised trial evaluating RUTFs with energy-rich and nutrient-rich home foods for the management of children with SAM without complications. 
Table 3 Primary and secondary outcomes in the treatment phase

\begin{tabular}{|c|c|c|c|c|c|}
\hline & \multicolumn{3}{|l|}{ Treatment groups } & \multicolumn{2}{|l|}{$\begin{array}{l}\text { Adjusted OR* } 95 \% \mathrm{Cl} \\
\text { p Value between }\end{array}$} \\
\hline & $\begin{array}{l}\text { A-HPF } \\
n=285\end{array}$ & $\begin{array}{l}\text { RUTF-C } \\
\mathrm{n}=\mathbf{2 8 0}\end{array}$ & $\begin{array}{l}\text { RUTF-L } \\
\mathrm{n}=290\end{array}$ & RUTF-C and A-HPF & RUTF-L and A-HPF \\
\hline \multicolumn{6}{|l|}{ Primary outcome } \\
\hline $\begin{array}{l}\text { Recovered by } \\
16 \text { weeks }\end{array}$ & $122(42.8)$ & $133(47.5)$ & $165(56.9)$ & $\begin{array}{l}1.28(0.90 \text { to } 1.82) \\
p=0.164\end{array}$ & $\begin{array}{l}1.71(1.20 \text { to } 2.43) \\
p=0.003\end{array}$ \\
\hline \multicolumn{4}{|c|}{ Weight gain in $\mathrm{g} / \mathrm{kg} /$ day of baseline weight } & \multicolumn{2}{|l|}{ Adjusted difference } \\
\hline Mean (SD) & $2.64(3.47)$ & $3.05(3.41)$ & $3.52(3.92)$ & $\begin{array}{l}0.44(-0.16 \text { to } 1.02) \\
p=0.148\end{array}$ & $\begin{array}{l}0.89(0.30 \text { to } 1.48) \\
p=0.003\end{array}$ \\
\hline Median (IQR) & $1.30(0.80-3.02)$ & $1.61(0.86-4.03)$ & $1.96(0.93-4.77)$ & & \\
\hline \multicolumn{6}{|c|}{ Time to recovery (week) in children who recovered during the treatment phase } \\
\hline \multirow[t]{2}{*}{ Children died } & 0 & 2 & 1 & & \\
\hline & & & & Adjusted OR* $95 \% \mathrm{Cl}$ & \\
\hline \multirow[t]{2}{*}{$\begin{array}{l}\text { Children } \\
\text { hospitalised }\end{array}$} & $30(10.5)$ & $30(10.7)$ & $22(7.6)$ & $\begin{array}{l}0.96(0.55 \text { to } 1.67) \\
p=0.884\end{array}$ & $\begin{array}{l}0.72(0.40 \text { to } 1.31) \\
p=0.286\end{array}$ \\
\hline & $n=243$ & $n=235$ & $n=249$ & & \\
\hline $\begin{array}{l}\text { Children with } \\
\text { diarrhoeat at any } \\
\text { time during the } \\
\text { treatment phase }\end{array}$ & $101(41.6)$ & $92(39.2)$ & $109(43.8)$ & $\begin{array}{l}0.94(0.62 \text { to } 1.42) \\
p=0.773\end{array}$ & $\begin{array}{l}1.19(0.79 \text { to } 1.78) \\
p=0.413\end{array}$ \\
\hline $\begin{array}{l}\text { Children with ALRI } \\
\text { at any time during } \\
\text { the treatment phase }\end{array}$ & $38(15.6)$ & $24(10.2)$ & $35(14.1)$ & $\begin{array}{l}0.59(0.34 \text { to } 1.04) \\
p=0.069\end{array}$ & $\begin{array}{l}0.84(0.50 \text { to } 1.41) \\
p=0.506\end{array}$ \\
\hline
\end{tabular}

All values are $\mathrm{n}(\%)$ unless otherwise indicated.

${ }^{*}$ Generalised Linear Model (GLM) model sex, WHZ at enrolment, family structure, age at enrolment, maternal education, religion, caste, site and peer support.

†Diarrhoea: passage of $\geq 3$ loose or watery stools in a 24-hour period.

$\ddagger A L R I$ : presence of cough or difficult breathing and either fast breathing or lower chest indrawing.

$\S$ Fever as reported by caregiver.

A-HPF, micronutrient-enriched (augmented) energy-dense home-prepared foods; ALRI, acute lower respiratory tract infection; RUTF-C, centrally produced ready-to-use therapeutic food; RUTF-L, locally prepared ready-to-use therapeutic food; WHZ, weight-for-height Z-score.

Our main finding is that in an efficacy study, locally produced RUTF is superior to A-HPF in achieving recovery. Our study confirms the findings of quasi-randomised trials in Malawi on the efficacy of RUTF compared with standard diets for home management of children with uncomplicated SAM. ${ }^{12} 1525$

In this trial, about half the children recovered with a package of interventions that included diets of high nutritional value provided free of cost, peer support for feeding, antibiotics at the initiation of treatment and increased access to healthcare for morbidity.

\section{Comparison with other studies and interpretation}

The recovery rates are lower than those observed in Africa, despite a longer duration of treatment and support to families for feeding. One of the reasons for the lower recovery rates may be that almost all children in our study had marasmus. In similar studies in Africa, the majority of children had kwashiorkor, and those with kwashiorkor had higher recovery rates compared with those with marasmus. ${ }^{24} 2533$ The prevalence of oedema in children with SAM in African studies ranged from $39 \%$ to $80 \%$ but was only $0.2 \%$ in our trial. The mean WHZ was also lower in our study compared with the African studies (detailed information in online supplementary file)..$^{12} 152426$ The evidence suggests that children with kwashiorkor tend to have higher weight-for-age than those with marasmus and may need to gain less weight to recover once they no longer have oedema. Diarrhoea impairs weight and height gain and thus leads to lower recovery rates. ${ }^{34} 35$ Infection also causes malnutrition due to deterioration of immune 
Figure 2 Time to recovery in the three treatment groups. A-HPF, micronutrient-enriched (augmented) energy-dense home-prepared foods; RUTF-C, centrally produced ready-to-use therapeutic food; RUTF-L, locally prepared ready-to-use therapeutic food.

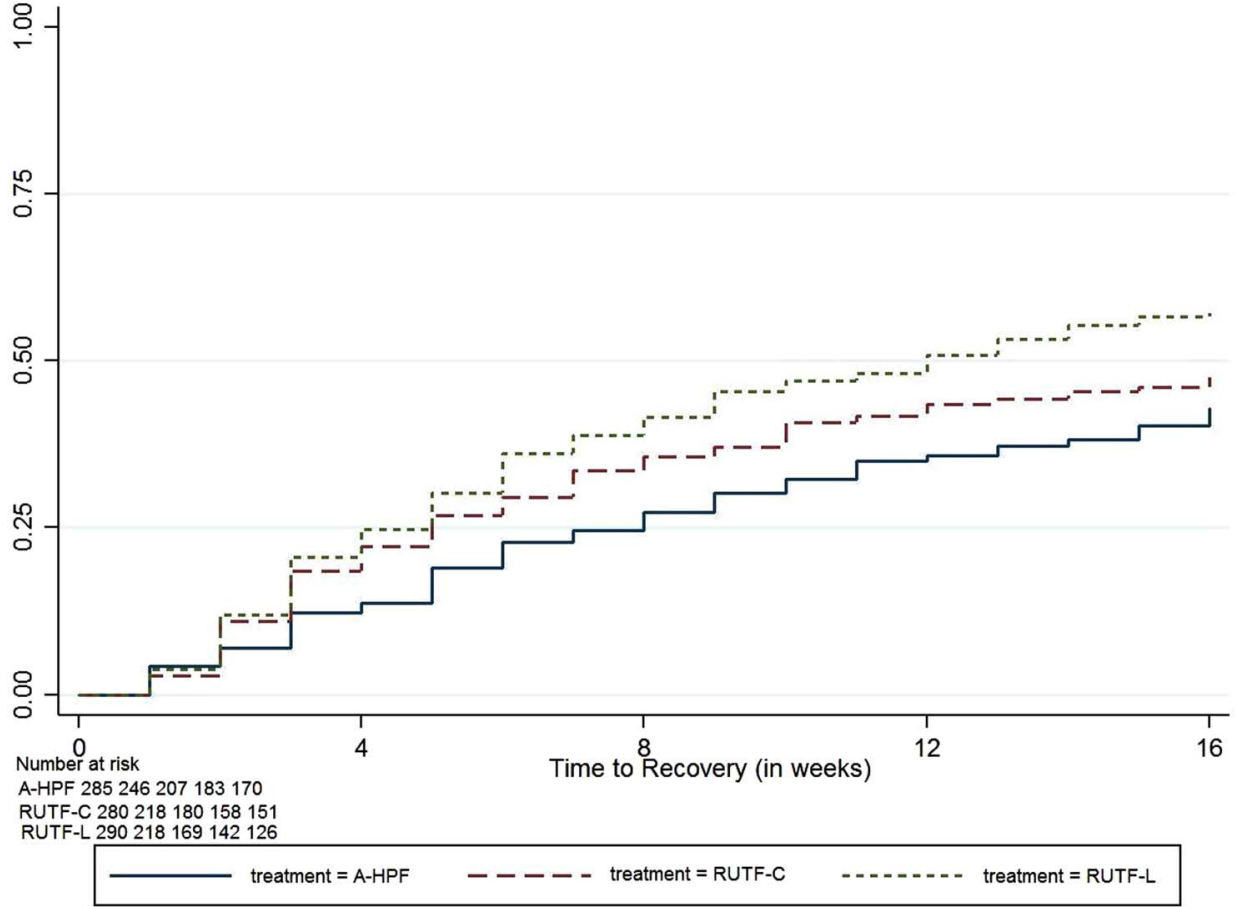

function which increases susceptibility to infection and metabolic responses that impair nutritional status. ${ }^{36}$ Further, around half the mothers of enrolled children were themselves malnourished; this may have resulted in less energetic efforts at feeding their malnourished children. Maternal underweight is one of the risk factors for childhood malnutrition and is closely associated with fetal, child and adolescent undernutrition. ${ }^{37}$

An additional explanation for the differences is that most African studies used height at enrolment to calculate WHZ during the follow-up period. We instead used subsequent height, measured concurrently with weights. In our study, height and weight were measured together every week during the follow-up period. Using height at enrolment increases the proportion of children who reach the cut-off for recovery.

The average weight gain in our trial was $3.5 \mathrm{~g} / \mathrm{kg} /$ day. This was considerably lower than $9.4 \mathrm{~g} / \mathrm{kg} /$ day observed in a study in Central India but closer to the observed in a community-based management of SAM project in Bihar that reported a weight gain of $4.9 \mathrm{~g} / \mathrm{kg} /$ day, possibly overestimated due to exclusion of defaulters $(38 \%) .{ }^{38}{ }^{39}$ In another trial from North India, the recovery rate (defined as gaining $115 \%$ of baseline weight) was $46 \%$ and the weight gain was $3 \mathrm{~g} / \mathrm{kg} /$ day, both similar to our study. ${ }^{40}$

Important lessons were gained from study implementation. The most valuable lesson was that in addition to the type of diet, extra support for feeding seems important in our setting for weight gain and recovery. Help from local experienced women seemed to improve food intake. It is likely that peer supporters, in addition to practical help, enhanced engagement and skills of the mother, resulting in children eating more. ${ }^{41} 42$
The finding that many children remain or slide back into moderate or severe malnutrition as early as within 16 weeks after the end of treatment is important for programmes. It underscores the importance of adequately sustained nutrition support after RUTF treatment is stopped. While linkages between families and the government anganwadi centres that provide supplementary nutrition services were established, additional measures may be required to sustain the improvements from the treatment phase. These may include close monitoring, improved counselling, support to caregivers, provision of additional food supplements, including giving RUTF for a longer period, and prompt treatment of illnesses. A recent trial in moderately malnourished Malawian children found that those treated with supplementary foods for 12 weeks had lower relapse rates and remained well nourished during the subsequent follow-up period of 12 months. ${ }^{43}$ Such longer period of care is more likely to be feasible in settings where management of children with SAM is embedded within efforts to prevent malnutrition.

\section{Strengths and weaknesses of the study}

Our study was a multicentre randomised trial conducted in different regions of India covering diverse populations. The community-based identification of SAM contributed to greater generalisability. Rigorous supervision and control of intervention delivery ensured uninterrupted food supplies across all the groups. Sharing of ingredients for preparation of foods at home is unlikely to have significantly reduced the amount of food offered to the child with SAM because supplies were provided in excess of the child's consumption and in-depth interviews with caregivers revealed little evidence of sharing. 
Table 4 Secondary outcomes during the sustenance phase (16 weeks after the end of the treatment phase)

\begin{tabular}{|c|c|c|c|c|c|}
\hline & \multirow[b]{2}{*}{$\begin{array}{l}\text { A-HPF } \\
n=281^{*}\end{array}$} & \multirow[b]{2}{*}{$\begin{array}{l}\text { RUTF-C } \\
n=273 \dagger\end{array}$} & \multirow[b]{2}{*}{$\begin{array}{l}\text { RUTF-L } \\
n=284 \ddagger\end{array}$} & \multicolumn{2}{|l|}{$\begin{array}{l}\text { Adjusted OR } 95 \% \text { CI } \\
\text { p Value between }\end{array}$} \\
\hline & & & & RUTF-C and A-HPF & RUTF-L and A-HPF \\
\hline \multicolumn{6}{|l|}{ At the end of the sustenance phase } \\
\hline$W H Z \geq-2$ (met definition of 'recovery') & $41(14.6)$ & $33(12.1)$ & $49(17.3)$ & $\begin{array}{l}0.78(0.47 \text { to } 1.34) \\
p=0.392\end{array}$ & $\begin{array}{l}1.12(0.74 \text { to } 1.95) \\
p=0.464\end{array}$ \\
\hline WHZ $<-3$ (severe acute malnutrition) & $119(42.4)$ & $111(40.7)$ & $83(29.2)$ & $\begin{array}{l}0.87(0.61 \text { to } 1.26) \\
p=0.470\end{array}$ & $\begin{array}{l}0.58(0.40 \text { to } 0.85) \\
p=0.005\end{array}$ \\
\hline \multicolumn{6}{|c|}{ Change between enrolment and the end of the sustenance phase } \\
\hline & & & & \multicolumn{2}{|c|}{ Adjusted difference $95 \% \mathrm{Cl}$} \\
\hline $\begin{array}{l}\text { Mid-upper arm circumference, } \\
\text { mean (SD) }\end{array}$ & $0.75(0.68)$ & $0.68(0.72)$ & $0.81(0.75)$ & $\begin{array}{l}-0.06(-0.17 \text { to } 0.04) \\
p=0.226\end{array}$ & $\begin{array}{l}0.08(-0.02 \text { to } 0.19) \\
p=0.115\end{array}$ \\
\hline
\end{tabular}

Three children (two in the RUTF-C group and one in the RUTF-L group) died during the treatment phase and two children (one each in the RUTF-C and RUTF-L groups) died during the sustenance phase. All values are $n(\%)$ unless otherwise indicated.

*Of the 301 enrolled children, 285 completed the treatment phase and of them 281 completed the sustenance phase. Reasons for loss: refusal $(n=3)$, moved away $(n=1)$.

tOf the 298 enrolled children, 280 completed the treatment phase and of them 273 completed the sustenance phase. Reasons for loss: moved away $(n=3)$, clinically diagnosed allergy $(n=1)$, died during the treatment phase $(n=2)$ and died during the sustenance phase $(n=1)$.

$\ddagger$ Of the 307 enrolled children, 290 infants completed the treatment phase and of them 284 completed the sustenance phase. Reasons for loss: refusal $(n=2)$, moved away $(n=2)$, died during the treatment phase $(n=1)$ and died during the sustenance phase $(n=1)$.

$\S G L M$ model adjusted for all potential covariates as sex, WHZ at enrolment, family structure, age at enrolment, maternal education, religion, caste, site and peer support.

A-HPF, micronutrient-enriched (augmented) energy-dense home-prepared foods; RUTF-C, centrally produced ready-to-use therapeutic food;

RUTF-L, locally prepared ready-to-use therapeutic food; WHZ, weight-for-height Z-score.

The outcome measurement team was independent from the intervention delivery team and workers underwent periodic standardisation exercises to ensure quality of anthropometric data.

Our study results also make an additional contribution to the literature because in the past children with SAM have been treated for 8 weeks. Continuing treatment for 16 weeks provides added value in this context where recovery rates were lower than expected at 8 weeks.

The study had some limitations. Around $25 \%$ of the children with MUAC $<13 \mathrm{~cm}$ did not come to the study clinic. This might have affected generalisability. This, however, should not have affected the internal validity as children were only randomised after the confirmation of measurements at the clinic. Blinding could have further reduced the risk of bias but this was not feasible as the three interventions had visibly different characteristicsone was provided in packets, the other in jars and the third comprised of food ingredients. Based on findings from the initial months, counselling was intensified in all three groups through peer supporters. Therefore, children enrolled earlier received less intensive counselling and help than those enrolled later in the trial. The overall recovery rates we reported are therefore likely to be conservative. We lacked a reliable measure for consumption in the group given ingredients to prepare food at home (A-HPF) due to the complexity of obtaining valid quantitative recalls for the recipes actually used.

Over $40 \%$ of children did not recover after RUTF use for 16 weeks. Since the therapeutic foods were discontinued as soon as the child met the definition of recovery, even a small deterioration in WHZ would mean that the child no longer met the definition for recovery by the time of the measurement 16 weeks later.

\section{Implications}

Our study shows that home-based management of children with uncomplicated SAM is an effective and feasible option and that use of a RUTF-L results in higher recovery rates than feeding nutrient-dense and caloriedense home foods. The gains observed during the initial 16 weeks, however, decline after treatment. Other approaches need to be considered to improve long-term outcomes including prolonged use of a RUTF-L. Setting up local units for production of RUTF does not require huge investment in terms of equipment, infrastructure or personnel. The procedures for production are systematic and simple to replicate.

Despite the effectiveness of home-based management, additional or alternative approaches are needed for the relatively large proportion of children with 
uncomplicated SAM who do not recover after treatment for 16 weeks.

Our findings indicate that rethinking about ways to combat the problem of severe malnutrition in children are required.

\author{
Author affiliations \\ ${ }^{1}$ Centre for Health Research and Development, Society for Applied Studies, \\ New Delhi, India \\ ${ }^{2}$ Christian Medical College, Vellore, Tamil Nadu, India \\ ${ }^{3}$ Action Research and Training for Health, Udaipur, Rajasthan, India \\ ${ }^{4}$ Sitaram Bhartia Institute of Science and Research, New Delhi, India \\ ${ }^{5}$ Department of Maternal, Newborn, Child and Adolescent Health, World \\ Health Organisation, Geneva, Switzerland \\ ${ }^{6}$ Centre for Intervention Science in Maternal and Child Health, Centre for \\ International Health, University of Bergen, Bergen, Norway
}

\section{Handling editor Valery Ridde}

Acknowledgements The authors acknowledge the contribution and support of families of participating children, the community members who served as peer supporters and others in the community who supported this work in other ways. The authors are grateful to the National Alliance for Research for SAM (chair Dr MK Bhan) and its secretariat (Clinical Development Services Agency) from the Department of Biotechnology, Ministry of Health, Indian Council of Medical Research, and public health experts, for their ongoing review of the study and constructive suggestions. The authors are also thankful to Mercedes de Onis, Department of Nutrition (WHO, Geneva) for helping set up the standardisation of anthropometric measurements including development of a manual for the same. They are also grateful to Zachary Linneman for the guidance on setting up local RUTF production units at the three trial sites and assistance in developing the manual of procedures. The authors acknowledge the DSMB members-Dr Vinod Paul (Chair), Dr Allan Donner, Dr Sunil Sazawal, Dr Panna Choudhury (deceased September 2015), Dr AM Khan and Dr RM Pandey. The authors are thankful to all the collaborating hospitals where the study participants were referred for management. The Society for Applied Studies acknowledges the core support provided by the Department of Maternal, Newborn, Child and Adolescent Health, WHO (Geneva); and the Centre for Intervention Science in Maternal and Child Health (RCN Project No. 223269), which is funded by the Research Council of Norway through its Centres of Excellence scheme and the University of Bergen (UiB), Norway. The authors also acknowledge the support extended by the Knowledge Integration and Technology Platform (KnIT), a Grand Challenges Initiative of the Department of Biotechnology and Biotechnology Industry Research Assistance Council (BIRAC) of Government of India and Bill \& Melinda Gates Foundation (USA).

Collaborators Other members of the Study Group. SAS: Sowmya Prakash, Rimpi Kaushik, Gunjan Aggarwal, Rajkumari Suchitra, Priti Sharma. CMC: Kuryan George, Jasmine Helan Prasad, Venkatesan Sankarapandian, Preethi Ragasudha, Dulari Gupta. ARTH: Anandilal Sharma, Anjana Verma, Ashutosh Sharma, Trupti Patel, Priya Krishnan, Satyanarayan Panchal, Hitesh Rawal. Coordination Unit. Kiran Bhatia, Girish Chand Pant, Medha Shekhar.

Contributors All authors contributed substantially to the design and undertaking of the study, its analyses and writing of the manuscript. NB, SBM, $A B, S D I, J M$ and RB designed the study. NB and ST coordinated the study and did data management and analysis, with technical support from RB, JM and SY. The following were responsible for the day-to-day implementation: SBM, SM and HSS (Delhi); AB, RAP and VRM (Vellore); and SDI, KI and VS (Udaipur). The study results were interpreted and the manuscript was prepared jointly during a workshop and has the final approval of all authors. NB affirms that the manuscript is an honest, accurate and transparent account of the study; that no important aspects of the study have been omitted; and that any discrepancies from the study as planned have been explained.

Funding The trial was funded by the Bill \& Melinda Gates Foundation (grant number OPP1033634). The corresponding author had full access to all the data in the study and had final responsibility for the decision to submit the publication.
Disclaimer The opinions expressed by them in this paper are their own and do not necessarily reflect the policy of the WHO.

Competing interests All authors have completed the Unified Competing Interest Form at http://www.icmje.org/coi_disclosure.pdf (available on request from the corresponding author) and declare that RB and SY are staff members of the WHO.

Ethics approval Society for Applied Studies, New Delhi; Christian Medical College, Vellore; Action Research and Training for Health, Udaipur; and the WHO Ethics Review Committee.

Provenance and peer review Not commissioned; externally peer reviewed.

Data sharing statement The authors have a data sharing agreement between the participating sites and consent for data sharing was obtained from patients at recruitment. Reasonable requests for patient-level data should be made to the corresponding author and will be considered by the National Research Alliance for SAM that provided oversight to the study. The presented data are anonymised and the risk of identification is low.

Open Access This is an Open Access article distributed in accordance with the Creative Commons Attribution Non Commercial (CC BY-NC 4.0) license, which permits others to distribute, remix, adapt, build upon this work noncommercially, and license their derivative works on different terms, provided the original work is properly cited and the use is non-commercial. See: http:// creativecommons.org/licenses/by-nc/4.0/

\section{REFERENCES}

1. Olofin I, McDonald CM, Ezzati M, et al. Associations of suboptimal growth with all-cause and cause-specific mortality in children under five years: a pooled analysis of ten prospective studies. PLOS ONE 2013;8:e64636.

2. Ahmed T, Hossain M, Mahfuz M, et al. Severe acute malnutrition in Asia. Food Nutr Bull 2014;35(2 Suppl):S14-26.

3. Ministry of Women and Child Development, Government of India. Rapid Survey on Children, 2013-2014: India Fact Sheet. http://wcd. nic.in/issnip/National_Fact\%20sheet_RSOC\%20_02-07-2015.pdf

4. World Health Organization. Joint Child Malnutrition Estimates (UNICEF-WHO-WB): Regional estimates for prevalence and number affected for wasting and severe wasting (2014). http://apps.who.int/ gho/data/view.wrapper.nutrition-1-5?lang=en\&showonly=nutrition

5. Bhutta ZA, Das JK, Rizvi A, et al. Evidence-based interventions for improvement of maternal and child nutrition: what can be done and at what cost? Lancet 2013;382:452-77.

6. Hossain MM, Hassan MQ, Rahman MH, et al. Hospital management of severely malnourished children: comparison of locally adapted protocol with WHO protocol. Indian Pediatr 2009;46:213-17.

7. Khanum S, Ashworth A, Huttly SR. Growth, morbidity, and mortality of children in Dhaka after treatment for severe malnutrition: a prospective study. Am J Clin Nutr 1998;67:940-5.

8. Collins S, Dent N, Binns P, et al. Management of severe acute malnutrition in children. Lancet 2006;368:1992-2000.

9. Sadler K, Myatt M, Feleke T, et al. A comparison of the programme coverage of two therapeutic feeding interventions implemented in neighbouring districts of Malawi. Public Health Nutr 2007;10:907-13.

10. Khanum S, Ashworth A, Huttly SR. Controlled trial of three approaches to the treatment of severe malnutrition. Lancet 1994;344:1728-32.

11. Briend A, Lacsala R, Prudhon $C$, et al. Ready-to-use therapeutic food for treatment of marasmus. Lancet 1999;353:1767-8.

12. Ciliberto MA, Sandige H, Ndekha MJ, et al. Comparison of home-based therapy with ready-to-use therapeutic food with standard therapy in the treatment of malnourished Malawian children: a controlled, clinical effectiveness trial. Am J Clin Nutr 2005;81:864-70.

13. Collins $\mathrm{S}$. Treating severe acute malnutrition seriously. Arch Dis Child 2007;92:453-61.

14. Diop el HI, Dossou NI, Ndour MM, et al. Comparison of the efficacy of a solid ready-to-use food and a liquid, milk-based diet for the rehabilitation of severely malnourished children: a randomized trial. Am J Clin Nutr 2003;78:302-7.

15. Manary MJ, Ndkeha MJ, Ashorn P, et al. Home based therapy for severe malnutrition with ready-to-use food. Arch Dis Child 2004:89:557-61.

16. Sandige $\mathrm{H}$, Ndekha MJ, Briend $\mathrm{A}$, et al. Home-based treatment of malnourished Malawian children with locally produced or imported ready-to-use food. J Pediatr Gastroenterol Nutr 2004;39:141-6. 
17. World Health Organization. Community- based management of severe acute malnutrition: a joint statement by the World Health Organization, the World Food Programme, the United Nations System Standing Committee on Nutrition and the United Nations Children's Fund. Geneva: World Health Organization, 2007.

18. Kin LH. The effectiveness of the use of "ready-to-use" therapeutic food (RUTF) in treating malnourished children in developing countries and the way forward. University of Hong Kong, 2013.

19. Kapil U. Ready to use therapeutic food (RUTF) in the management of severe acute malnutrition in India. Indian Pediatr 2009;46:381-2.

20. Bhutta ZA, Shekar M, Ahmed T. Mainstreaming interventions in the health sector to address maternal and child undernutrition. Matern Child Nutr 2008;4(Suppl 1):1-4.

21. Schoonees A, Lombard M, Musekiwa A, et al. Ready-to-use therapeutic food for home-based treatment of severe acute malnutrition in children from six months to five years of age. Cochrane Database Syst Rev 2013;6:CD009000.

22. Gupta P, Shah D, Sachdev HP, et al. National workshop on "development of guidelines for effective home based care and treatment of children suffering from severe acute malnutrition". Indian Pediatr 2006;43:131-9.

23. World Health Organization. Guideline: updates on the management of severe acute malnutrition in infants and children. Geneva: World Health Organization, 2013.

24. Oakley E, Reinking J, Sandige $\mathrm{H}$, et al. A ready-to-use therapeutic food containing $10 \%$ milk is less effective than one with $25 \%$ milk in the treatment of severely malnourished children. $J$ Nutr 2010;140:2248-52.

25. Linneman Z, Matilsky D, Ndekha M, et al. A large-scale operational study of home-based therapy with ready-to-use therapeutic food in childhood malnutrition in Malawi. Matern Child Nutr 2007;3:206-15.

26. Ndekha MJ, Manary MJ, Ashorn P, et al. Home-based therapy with ready-to-use therapeutic food is of benefit to malnourished, HIV-infected Malawian children. Acta Paediatr 2005;94:222-5.

27. WHO Multicentre Growth Reference Study Group. WHO Child Growth Standards based on length/height, weight and age. Acta Paediatr Suppl 2006;450:76-85.

28. Government of India. Integrated Management of Neonatal and Childhood Illness. http://nrhm.gov.in/nrhm-components/rmnch-a/ child-health-immunization/child-health/guidelines.html

29. Manary MJ. Local production and provision of ready-to-use therapeutic food (RUTF) spread for the treatment of severe childhood malnutrition. Food Nutr Bull 2006;27(3 Suppl):S83-9.
30. Ministry of Health and Family Welfare, Government of India. National Rural Health Mission: About Accredited Social Health Activist. http:// nrhm.gov.in/communitisation/asha/about-asha.html

31. Ministry of Women and Child Development, Government of India Integrated Child Development Services Scheme. http://icds-wcd.nic. in/icds/icds.aspx

32. de Onis M, Onyango AW, Van den Broeck J, et al. Measurement and standardization protocols for anthropometry used in the construction of a new international growth reference. Food Nutr Bull 2004;25(1 Suppl):S27-36.

33. Trehan I, Goldbach HS, LaGrone LN, et al. Antibiotics as part of the management of severe acute malnutrition. $N$ Engl J Med 2013:368:425-35.

34. Guerrant RL, Carneiro-Filho BA, Dillingham RA. Cholera, diarrhea, and oral rehydration therapy: triumph and indictment. Clin Infect Dis 2003;37:398-405

35. Guerrant RL, Schorling JB, McAuliffe JF, et al. Diarrhea as a cause and an effect of malnutrition: diarrhea prevents catch-up growth and malnutrition increases diarrhea frequency and duration. Am J Trop Med Hyg 1992;47(Pt 2):28-35.

36. Rodriguez L, Cervantes E, Ortiz R. Malnutrition and gastrointestinal and respiratory infections in children: a public health problem. Int J Environ Res Public Health 2011;8:1174-205.

37. Bhutta ZA. What does India need to do to address childhood malnutrition at scale? Soc Sci Med 2016;157:186-8

38. Burza S, Mahajan R, Marino E, et al. Community-based management of severe acute malnutrition in India: new evidence from Bihar. Am J Clin Nutr 2015;101:847-59.

39. Thakur GS, Singh HP, Patel C. Locally-prepared ready-to-use therapeutic food for children with severe acute malnutrition: a controlled trial. Indian Pediatr 2013;50:295-9.

40. Shewade HD, Patro BK, Bharti B, et al. Effectiveness of indigenous ready-to-use therapeutic food in community-based management of uncomplicated severe acute malnutrition: a randomized controlled trial from India. J Trop Pediatr 2013;59:393-8.

41. Dearden KA, Hilton S, Bentley ME, et al. Caregiver verbal encouragement increases food acceptance among Vietnamese toddlers. J Nutr 2009;139:1387-92.

42. Vazir S, Engle P, Balakrishna N, et al. Cluster-randomized trial on complementary and responsive feeding education to caregivers found improved dietary intake, growth and development among rura Indian toddlers. Matern Child Nutr 2013;9:99-117.

43. Trehan I, Banerjee S, Murray E, et al. Extending supplementary feeding for children younger than 5 years with moderate acute malnutrition leads to lower relapse rates. J Pediatr Gastroenterol Nutr 2015;60:544-9. 